August 2000

\title{
Lumps in level truncated open string field theory
}

\author{
Robert de Mello Koch and João P. Rodrigues \\ Department of Physics and Center for Nonlinear Studies, \\ University of the Witwatersrand, \\ Wits, 2050, South Africa \\ robert,joao@physnet.phys.wits.ac.za
}

\begin{abstract}
The ratios of the masses of $D(p-d)$ branes to the masses of $D p$ in open bosonic string field theory are computed within the modified level truncation approximation of Moeller, Sen and Zweibach. At the lowest non-trivial truncation requiring the addition of new primary states, we find evidence of rapid convergence to the expected result for $2 \leq d \leq 6$ providing additional evidence for the consistency of this approximation.
\end{abstract}


There is growing evidence that open string field theory provides a direct approach to study string theory tachyons. This recent progress has been possible as a result of Sen's conjecture that there is an extremum of the tachyon potential at which the total negative potential energy exactly cancels the tension of the $D$-brane[1] and that lump solutions are identified with lower dimensional branes [1,2]. These conjectures have been extended for tachyons living on coincident $D$-brane anti- $D$-brane pairs and for tachyons on the non-BPS $D$-branes of type IIA or IIB superstring theories [3, 4, 5, 6, 7, 8]. These conjectures provide precise predictions which can be used to test and develop approximation techniques in open string field theory. The results obtained thus far are impressive [9, 10, 11, 12, 13, 14, 15, 16, 17, 18, 19, 20]. In particular, the level truncation approximation has proved to be powerful in this context. The original argument for level truncation appeared in the unpublished work[21] and was subsequently used by Samuel and Kostelecky[22] to study the vacuum structure of string field theory. In this article, we are interested in a variant of the level truncation scheme, introduced by Moeller, Sen and Zweibach (MSZ) [18]. Within this scheme, MSZ were able to compute the ratio of the mass of a $D(p-1)$-brane to a $D p$-brane to an impressive accuracy of about $1 \%$ !

This ratio was computed in [12] in the field theory limit and good agreement with the expected result was obtained. Indeed, the field theory lump reproduces $78 \%$ of the expected $D(p-1)$ brane tension. Including the stringy corrections from the momentum dependence of the interaction terms does not significantly change the tension of the lump. This is not the case for the ratios of the masses of $D(p-d)$ branes to the masses of $D p$ branes for $d>1$. As $d$ is increased the field theory predictions get worse and for $d$ large enough there are no lump solutions. When the stringy corrections are included, lump solutions can be found for any value of $d[13]$. However, initial studies of these ratios show that the leading order in the level truncation approximation over estimates this ratio for $d>4$, with the results becoming increasingly worse as $d$ is increased. As a further interesting check of the modified level truncation scheme, one could compute the ratios of the masses of $D(p-d)$ branes to the mass of a $D p$ brane. These ratios are more difficult to reproduce and obtaining them within the modified level truncation approximation will give important insight into how fast the approximation converges. This is the question that is studied in this article.

1 By turning on a large $B$ field the description of tachyon condensation can be drastically simplified. In this limit, the tension computed from tachyonic solitons exactly agree with the expected $D$-brane tensions 23]. 
The modified level truncation approximation starts by assigning to a state $\left|\Phi_{i}\right\rangle$, with number eigenvalue $N_{i}$, the level

$$
l\left(\Phi_{i, n}\right) \equiv \frac{\vec{n} \cdot \vec{n}}{R^{2}}+N_{i}-N_{0},
$$

where $N_{0}$ is the number eigenvalue for the zero momentum tachyon 18 . The level $(M, N)$ approximation to the action is then defined by keeping only fields with level $\leq M$ and terms in the action for which the sum of levels is $\leq N$. We assume that the background spacetime is the product of a $(d+1)+1$ dimensional flat spacetime labelled by the spacelike coordinates $\left(x^{1}, \ldots, x^{d}, y\right)$ and a timelike coordinate $x^{0}$, and a Euclidean manifold $\mathcal{M}$ described by a conformal field theory of central charge $26-d-2$. The spatial direction $y$ is non-compact; the $x^{i}, i=1, \ldots, d$ parametrize a torus $T^{d}: x^{i} \sim x^{i}+R$. As in [24 by studying the motion of the brane along this non-compact direction, we can compute its tension. For an open string ending on the $D$-brane, we put Neumann boundary conditions on the fields $\left(X^{1}, \ldots, X^{d}\right)$ and $X^{0}$, and Dirichlet boundary conditions on the field $Y$ and on the fields associated with the coordinates of $\mathcal{M}$. These boundary conditions are correct for a $D d$-brane wrapped on the $T^{d}$. Because the $D$-brane has a finite volume, it will have a finite mass.

The dynamics of the open strings with ends on this $D$-brane is described by the direct sum of the conformal field theories associated with the fields $X^{i}, Y, X^{0}$ and the manifold $\mathcal{M}$. Following [24] we will work on a subspace of the full string field theory Fock space. Towards this end, we denote the conformal field of the $X^{i}$ by $\mathrm{CFT}\left(T^{d}\right)$ and the conformal field theory of the fields $Y, X^{0}$ and of the manifold $\mathcal{M}$ by $\mathrm{CFT}^{\prime}=\mathrm{CFT}(Y) \oplus \mathrm{CFT}\left(X^{0}\right) \oplus \mathrm{CFT}(\mathcal{M})$. The Virasoro generators of the system are given by $L_{n}=L_{n}^{\text {ghost }}+L_{n}^{T^{d}}+L_{n}^{\prime}$, where $L_{n}^{\text {ghost }}$ are the Virasoro generators of the ghost system, $L_{n}^{T^{d}}$ are the Virasoro generators of $\mathrm{CFT}\left(T^{d}\right)$ and $L_{n}^{\prime}$ are the Virasoro generators of $\mathrm{CFT}^{\prime}$. The subspace of the full string Fock space that we will focus on is most easily characterized by grouping states into Verma modules[24]. Each Verma module can be labelled by a primary state. The Verma module contains this primary state together will all states obtained by acting on this primary with the Virasoro generators $L_{-n}^{T^{d}}, L_{-n}^{\prime}, c_{1}, c_{-n}$ and $b_{-n}$ with $n \geq 0$. Null states and their descendents should be removed. The truncation can now be described by specifying which primary states we consider. We consider primary states (with arbitrary momentum on the $T^{d}$ ) of $\mathrm{CFT}\left(T^{d}\right)$ which are even under $X \rightarrow-X$ and are trivial $\mathrm{CFT}^{\prime}$ primaries. We will also restrict to states of even twist. For more details, the reader should consult [24]. Working on this subspace was shown in 24, 18 to be a consistent truncation of the full open string field theory. 
If we work to a given level, then we need only consider the Verma modules built on a finite number of primary fields. In our subspace, the zero momentum tachyon mode is the only primary that needs to be considered at level 0 ; there are $d-1$ zero-momentum primaries to be considered at level 2 [25]. In the analysis of [18], the case $d=1$ was considered, so no new zero-momentum primaries had to be added until level 4 . Since we work only to level $(2,4)$ we need not consider any other zero-momentum primaries. Thus, the states that we consider are

$$
\begin{gathered}
\left|T_{\vec{n}}\right\rangle=c_{1} \cos \left(\frac{\vec{n} \cdot \vec{X}(0)}{R}\right)|0\rangle \\
\left|U_{\vec{n}}\right\rangle=c_{-1} \cos \left(\frac{\vec{n} \cdot \vec{X}(0)}{R}\right)|0\rangle \\
\left|V_{\vec{n}}\right\rangle=c_{1} L_{-2}^{T^{d}} \cos \left(\frac{\vec{n} \cdot \vec{X}(0)}{R}\right)|0\rangle, \\
\left|W_{\vec{n}}\right\rangle=c_{1} L_{-2}^{\prime} \cos \left(\frac{\vec{n} \cdot \vec{X}(0)}{R}\right)|0\rangle, \\
\left|Z_{\vec{n}}\right\rangle=c_{1} L_{-1}^{T^{d}} L_{-1}^{T^{d}} \cos \left(\frac{\vec{n} \cdot \vec{X}(0)}{R}\right)|0\rangle, \\
\left|S_{\vec{n}}^{i}\right\rangle=c_{1}\left(\alpha_{-1}^{1} \alpha_{-1}^{1}-\alpha_{-1}^{i+1} \alpha_{-1}^{i+1}-\alpha_{0}^{1} \alpha_{-2}^{1}+\alpha_{0}^{i+1} \alpha_{-2}^{i+1}\right) \cos \left(\frac{\vec{n} \cdot \vec{X}(0)}{R}\right)|0\rangle .
\end{gathered}
$$

The states $\left|S_{\overrightarrow{0}}^{i}\right\rangle$ are the new zero momentum primaries that need to be added at level 2 . We should not include the zero momentum $Z_{\overrightarrow{0}}$ mode, since it corresponds to the descendent of a null state. The index $i$ runs from $i=1, \ldots, d-1$. The conditions that the states $\left|S_{\vec{n}}^{i}\right\rangle$ are primary implies the following restriction on $\vec{n}$

$$
n^{1}= \pm n^{i+1}
$$

The string field is expanded in terms of these states as follows

$$
|\Phi\rangle=\sum_{\vec{n}}\left(t_{\vec{n}}\left|T_{\vec{n}}\right\rangle+u_{\vec{n}}\left|U_{\vec{n}}\right\rangle+v_{\vec{n}}\left|V_{\vec{n}}\right\rangle+w_{\vec{n}}\left|W_{\vec{n}}\right\rangle+z_{\vec{n}}\left|Z_{\vec{n}}\right\rangle+s_{\vec{n}}^{i}\left|S_{\vec{n}}^{i}\right\rangle\right) .
$$

To ensure that we have the $X^{i} \rightarrow-X^{i}$ symmetry we have to put restrictions on the coefficients appearing in this expansion. For example, in the case that $d=2$, states 
carrying momentum $\vec{n}=(1,1)$ are related to states carrying momentum $\vec{n}=(1,-1)$ by $X^{2} \rightarrow-X^{2}$. Thus, the coefficients corresponding to these states in (9) should be identified. If we were treating this problem in the $R \rightarrow \infty$ limit, we'd look for solutions which are rotationally invariant. However, for finite $R$, all that survives of the rotational invariance is a discrete subset of rotations which permute the different $X^{i}$. We will compute the potential below assuming this symmetry. Thus for example, in the case $d=2$, we will not distinguish between states carrying momentum $\vec{n}=(0,1)$ and states carrying momentum $\vec{n}=(1,0)$. In addition, we will not distinguish between $s_{0}^{i}$ for diferent values of $i$.

The string field theory action is given by

$$
S=\langle\Phi|Q| \Phi\rangle+\frac{g}{3}\left\langle V_{3}|| \Phi\right\rangle|\Phi\rangle|\Phi\rangle
$$

with $Q$ the first quantized BRST operator and $\left|V_{3}\right\rangle$ the three string interaction vertex. To compute the potential, we have used the operator representation of the vertex as an object in the three string (dual) Fock space 26]. Evaluation of the above two terms in the action then requires only algebraic manipulations. The details of this computation will appear elsewhere 25]. The result, up to level $(2,4)$ is (with $R=\sqrt{3}$ )

$$
\begin{gathered}
V(0,0)=-\frac{1}{2} t_{0}^{2}+\frac{1}{3} K^{3} t_{0}^{3} \\
V\left(\frac{1}{3}, \frac{2}{3}\right)=V(0,0)-\frac{d}{4}\left(1-\frac{1}{R^{2}}\right) t_{1}^{2}+\frac{d}{2} K^{3-\frac{2}{R^{2}}} t_{0} t_{1}^{2} \\
V\left(\frac{2}{3}, \frac{4}{3}\right)=V\left(\frac{1}{3}, \frac{2}{3}\right)+\frac{d(d-1)}{2} K^{3-\frac{4}{R^{2}}} t_{2}^{2} t_{0}-\frac{d(d-1)}{4}\left(1-\frac{2}{R^{2}}\right) t_{2}^{2}+\frac{d(d-1)}{2} K^{3-\frac{4}{R^{2}}} t_{1}^{2} t_{2} \\
V(1,2)=V\left(\frac{2}{3}, \frac{4}{3}\right)+d(d-1)(d-2)\left[\frac{1}{3} K^{3-\frac{6}{R^{2}}} t_{3}^{2} t_{0}-\frac{1}{6} t_{3}^{2}\left(1-\frac{3}{R^{2}}\right)\right. \\
V\left(\frac{4}{3}, \frac{8}{3}\right)=V(1,2)+d(d-1)(d-2)(d-3)\left[\frac{1}{6} \tilde{t}_{4}^{2} t_{0} K^{3-\frac{6}{R^{2}}} t_{1} t_{2} t_{3}+\frac{1}{3} K^{3-\frac{6}{R^{2}}} t_{2}^{3}\right] \\
\left.+\frac{1}{2} \tilde{t}_{4} t_{2}^{2} K^{3-\frac{8}{R^{2}}}+\frac{2}{3} t_{1} t_{3} \tilde{t}_{4} K^{3-\frac{8}{R^{2}}}+t_{3}^{2} t_{2} K^{3-\frac{8}{R^{2}}}\right]+d\left[\frac{1}{2} \tilde{t}_{4}^{2}\left(1-\frac{4}{R^{2}}\right)\right. \\
-\frac{\bar{t}_{4}^{2}}{4}\left(1-\frac{4}{R^{2}}\right)+\frac{d-1}{2} t_{2}^{2} \bar{t}_{4} K^{3-\frac{8}{R^{2}}}
\end{gathered}
$$




$$
\begin{aligned}
V\left(\frac{5}{3}, \frac{10}{3}\right) & =V\left(\frac{4}{3}, \frac{8}{3}\right)+d(d-1)\left(t_{0} \bar{t}_{5}^{2} K^{3-\frac{10}{R^{2}}}-\frac{1}{2} \bar{t}_{5}^{2}\left[1-\frac{5}{R^{2}}\right]+t_{1} \bar{t}_{4} \bar{t}_{5} K^{3-\frac{10}{R^{2}}}\right. \\
& \left.+2(d-2) t_{2} t_{3} \bar{t}_{5} K^{3-\frac{10}{R^{2}}}+\frac{1}{2}(d-2) t_{3}^{2} \bar{t}_{4} K^{3-\frac{10}{R^{2}}}\right)+d(d-1)(d-2)(d-3) \times \\
& \times(d-4)\left(\frac{1}{15} t_{0} \tilde{t}_{5}^{2} K^{3-\frac{10}{R^{2}}}-\frac{1}{30} \tilde{t}_{5}^{2}\left[1-\frac{5}{R^{2}}\right]+\frac{1}{3} t_{1} \tilde{t}_{4} \tilde{t}_{5} K^{3-\frac{10}{R^{2}}}+\frac{2}{3} t_{2} t_{3} \tilde{t}_{5} K^{3-\frac{10}{R^{2}}}\right. \\
& \left.+\frac{2}{3} t_{2} \tilde{t}_{4}^{2} K^{3-\frac{10}{R^{2}}}+t_{3}^{2} \tilde{t}_{4} K^{3-\frac{10}{R^{2}}}\right)+d(d-1) t_{1} t_{2} \bar{t}_{5} K^{3-\frac{8}{R^{2}}}
\end{aligned}
$$

$$
\begin{aligned}
& V(2,4)=V\left(\frac{5}{3}, \frac{10}{3}\right)+d(d-1)(d-2)\left(t_{0} \bar{t}_{6}^{2} K^{3-\frac{12}{R^{2}}}+2 t_{1} \bar{t}_{5} \bar{t}_{6} K^{3-\frac{12}{R^{2}}}+t_{2} \bar{t}_{5}^{2} K^{3-\frac{12}{R^{2}}}\right. \\
& +t_{1} t_{3} \bar{t}_{6} K^{3-\frac{10}{R^{2}}}+\frac{K^{1-\frac{6}{R^{2}}}}{3}\left[\frac{-5 d}{32}+\frac{3}{R^{2}}\right] t_{3}^{2} v_{0}+\frac{11}{48} K^{1-\frac{6}{R^{2}}} u_{0} t_{3}^{2}-\frac{5(26-d)}{96} K^{1-\frac{6}{R^{2}}} w_{0} t_{3}^{2} \\
& \left.-\frac{1}{2} \bar{t}_{6}^{2}\left[1-\frac{6}{R^{2}}\right]+t_{2} \bar{t}_{4} \bar{t}_{6} K^{3-\frac{12}{R^{2}}}\right)+d(d-1)(d-2)(d-3)(d-4)(d-5) K^{3-\frac{12}{R^{2}} \times} \\
& \left(\frac{1}{45} t_{0} \tilde{t}_{6}^{2}+\frac{2}{15} t_{1} \tilde{t}_{5} \tilde{t}_{6}+\frac{1}{3} t_{2} \tilde{t}_{4} \tilde{t}_{6}+\frac{1}{3} t_{2} \tilde{t}_{5}^{2}+\frac{2}{9} t_{3}^{2} \tilde{t}_{6}+\frac{4}{3} t_{3} \tilde{t}_{4} \tilde{t}_{5}+\frac{2}{3} \tilde{t}_{4}^{3}\right)+d(d-1) \times \\
& (d-2)(d-3) K^{3-\frac{12}{R^{2}}}\left(2 t_{3}^{2} \bar{t}_{6}+2 t_{3} \tilde{t}_{4} \bar{t}_{5}+\frac{1}{3} \tilde{t}_{4}^{2} \bar{t}_{4}\right)+\frac{19 K}{144} u_{0}^{2} t_{0}-\frac{5 d}{32} K t_{0}^{2} v_{0} \\
& -d(d-1)(d-2)(d-3)(d-4)(d-5) \frac{\tilde{t}_{6}^{2}}{90}\left(1-\frac{6}{R^{2}}\right)+d(d-1)\left(\frac{1}{4} s_{0}^{2}\right. \\
& \left.+\frac{1}{2} K^{1-\frac{4}{R^{2}}}\left[-\frac{5 d}{32}+\frac{2}{R^{2}}\right] t_{2}^{2} v_{0}+\frac{11}{32} K^{1-\frac{4}{R^{2}}} u_{0} t_{2}^{2}-\frac{5(26-d)}{64} K^{1-\frac{4}{R^{2}}} w_{0} t_{2}^{2}+\frac{32}{27} K s_{0}^{2} t_{0}\right) \\
& +\frac{K}{432}\left[\frac{25 d^{2}}{4}+128 d\right] t_{0} v_{0}^{2}+\frac{d}{2} K^{1-\frac{2}{R^{2}}}\left[-\frac{5 d}{32}+\frac{1}{R^{2}}\right] t_{1}^{2} v_{0}+\frac{11}{32} d K^{1-\frac{2}{R^{2}}} u_{0} t_{1}^{2} \\
& +\frac{11}{16} K t_{0}^{2} u_{0}-K \frac{5(26-d)}{32} t_{0}^{2} w_{0}+\frac{K}{432}\left[\frac{25(26-d)^{2}}{4}+128(26-d)\right] t_{0} w_{0}^{2}+\frac{d}{4} v_{0}^{2} \\
& -\frac{5(26-d)}{64} d K^{1-\frac{2}{R^{2}}} w_{0} t_{1}^{2}-\frac{55 d}{432} K t_{0} u_{0} v_{0}-\frac{55(26-d)}{432} K t_{0} u_{0} w_{0}-\frac{u_{0}^{2}}{2}+\frac{26-d}{4} w_{0}^{2} \\
& +\frac{25 d(26-d)}{864} K t_{0} w_{0} v_{0}+\frac{1}{2} d(d-1) t_{2} \bar{t}_{5}^{2} K^{3-\frac{12}{R^{2}}} \text {. }
\end{aligned}
$$

In this potential we have labelled the modes by their $\vec{n} \cdot \vec{n}$ eigenvalue. This labelling is not unique if $d>3$ and $\vec{n} \cdot \vec{n} \geq 4$. For example, when $d=4$ and $\vec{n} \cdot \vec{n}=4$ we can build the $\vec{n}$ as $\vec{n}=(1,1,1,1)$ or $\vec{n}=(2,0,0,0)$. We distinguish between these two vectors by using a tilde $(\tilde{t})$ to denote vectors whose only entries are 1 s and by using a bar $(\bar{t})$ to denote vectors whose entries include a 2 . This labeling is unique to the level considered here. We now seek lump solutions for the tachyon condensate which minimize this potential. 
The lump solutions are local minima of the potential. A direct minimization of the potential usually yields the tranlationally invariant vacuum which is the unique global minimum. To obtain the lump solutions, we found it simplest to work with the equations of motion. The numerical results obtained in this way were stable, both as $d$ is increased and as the level is increased. For example, the following table summarizes the values of the fields at the minimum corresponding to the $D 0$ lump, with $R=\sqrt{3}$ and $d=2$

\begin{tabular}{|r|r|r|r|r|r|}
\hline \hline & $\left(\frac{1}{3}, \frac{2}{3}\right)$ & $\left(\frac{2}{3}, \frac{4}{3}\right)$ & $\left(\frac{4}{3}, \frac{8}{3}\right)$ & $\left(\frac{5}{3}, \frac{10}{3}\right)$ & $(2,4)$ \\
\hline \hline$t_{0}$ & 0.1810 & 0.2863 & 0.3021 & 0.3186 & 0.3806 \\
\hline$t_{1}$ & 0.2435 & 0.2115 & 0.2025 & 0.1915 & 0.2127 \\
\hline$t_{2}$ & & -0.1253 & -0.1256 & -0.1257 & -0.1432 \\
\hline $\bar{t}_{4}$ & & & -0.0442 & -0.0495 & -0.0563 \\
\hline $\bar{t}_{5}$ & & & & 0.0280 & 0.0353 \\
\hline$u_{0}$ & & & & & 0.1268 \\
\hline$v_{0}$ & & & & & 0.0234 \\
\hline$w_{0}$ & & & & & 0.0417 \\
\hline$s_{0}^{i}$ & & & & & 0.00 \\
\hline \hline
\end{tabular}

The fact that $s_{0}^{i}$ is zero is a consequence of the fact that it enters the action quadratically. Generically, at this level, the potential would include terms linear in $s_{0}^{i}$ which would couple $s_{0}^{i}$ to the $t_{1}, t_{2}$ and $t_{3}$ tachyon modes. However, due the symmetry of the tachyon solution these couplings vanish once summed over momenta.

We have used these minima to compute the ratio of the mass of a $D(p-d)$-brane to the mass of a $D p$ brane. The quantity that we measure is

$$
r=\frac{E_{l u m p}}{T_{0}}=\frac{(2 \pi R)^{p}}{2^{p-1} \pi^{p-2}}\left(V_{l u m p}-V_{v a c}\right)
$$

where $E_{\text {lump }}$ in the energy of the lump solution, $T_{0}$ is the mass of a zero brane, $V_{\text {lump }}$ is the value of the potential evaluated at the lump solution and $V_{v a c}$ is the value of the potential evaluated at the global minimum corresponding to the translationally invariant tachyon condensate. The predicted value for this ratio is 1 . We have computed $V_{\text {lump }}$ and $V_{v a c}$ to the same order in the modified level truncation expansion. In 18 this parameter was called $r^{(2)}$. The values of $r$ obtained in this study are 


\begin{tabular}{|l|r|r|r|r|r|c|}
\hline \hline & $\left(\frac{1}{3}, \frac{2}{3}\right)$ & $\left(\frac{2}{3}, \frac{4}{3}\right)$ & $(1,2)$ & $\left(\frac{4}{3}, \frac{8}{3}\right)$ & $\left(\frac{5}{3}, \frac{10}{3}\right)$ & $(2,4)$ \\
\hline \hline$d=2$ & 1.3402 & 0.8992 & & 0.8377 & 0.7772 & 1.1303 \\
\hline$d=3$ & 2.3213 & 1.4237 & 1.0690 & 1.0278 & 0.9313 & 1.3277 \\
\hline$d=4$ & 4.0206 & 2.3658 & 1.5659 & 1.2712 & 1.1754 & 1.6384 \\
\hline$d=5$ & 6.963 & 4.009 & 2.472 & 1.7782 & 1.5036 & 2.0901 \\
\hline$d=6$ & 12.06 & 6.8225 & 4.0391 & 2.6862 & 2.0618 & 2.6641 \\
\hline \hline
\end{tabular}

The decrease in the value of $r$ as higher tachyon modes are added and the sharp increase in the tension at level $(2,4)$ qualitatively match the results obtained in [18].

For $d=2$ we have ploted the tachyon profile of the $D 0$ brane lump solution at levels $(2,4)$ and $(5 / 3,10 / 3)$

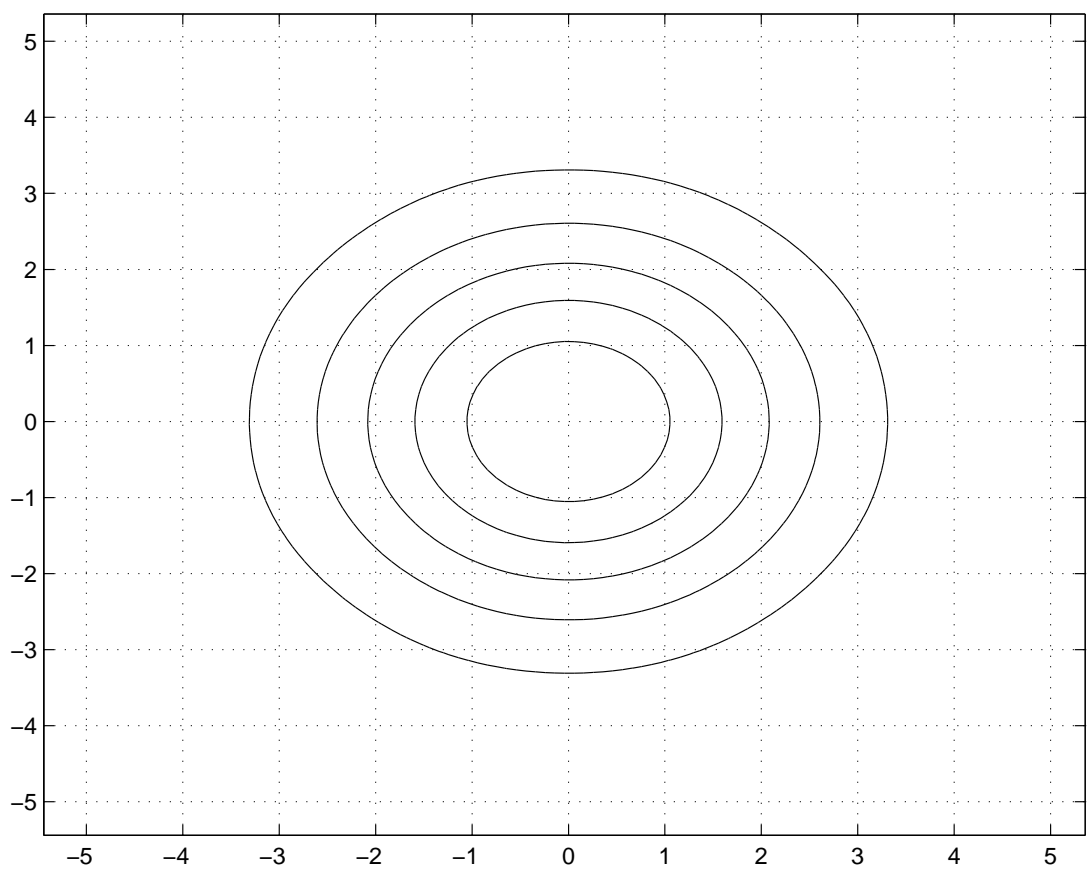

Fig. 1: A contour plot of the tachyon lump profile at level $(2,4)$ and at radius $R=\sqrt{3}$. We have reversed the signs of $t_{1}$ and $\bar{t}_{5}$, which shifts the lump's center from $x=y=\pi R$ to $x=y=0$. 


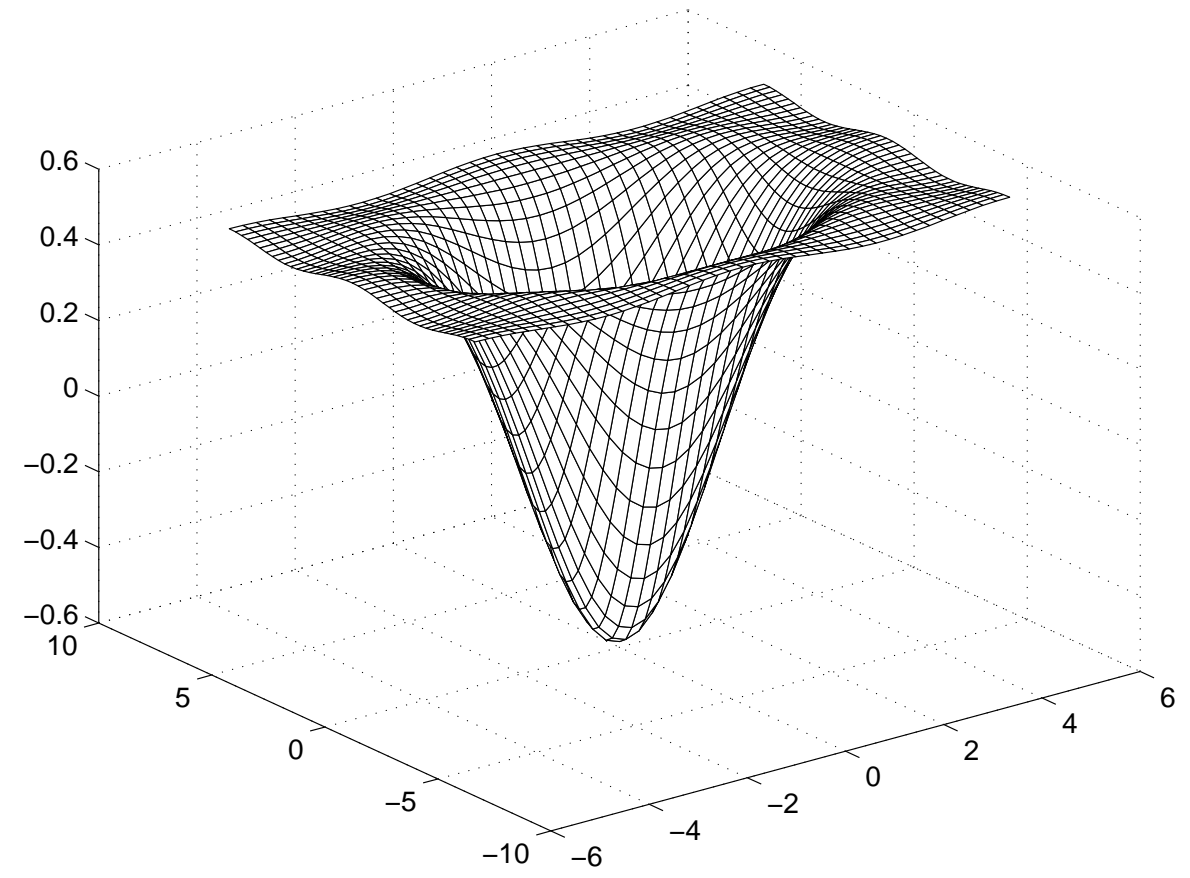

Fig. 2: A profile plot of the tachyon lump at level $\left(\frac{6}{3}, \frac{12}{3}\right)$ and at radius $R=\sqrt{3}$.

Our results indicate the the modified level truncation proposed by MSZ provides a powerful tool for the study of tachyon condensation in bosonic open string field theory. It is interesting to extend these results to higher level, to further test the accuracy and concergence of the modified level truncation approximation. It is possible to continue to level $(11 / 3,22 / 3)$ with $R=\sqrt{3}$ before we need to add any new zero-momentum primaries. This study is in progress.

Note Added: An independent study of two dimensional solitons in open bosonic string theory has recently appeared in [27]. The results of [27] agree with the results we obtained here for $d=2$.

Acknowledgements: We would like to thank Nicolas Moeller for helpful correspondence and detailed comparison. This was crucial for correcting a coefficient in equation (17). This research was supported in part by the NRF under Grant No. GUN-2034479. RdMK also thanks the University of the Witwatersrand for a Friedel Schellschop award. 


\section{References}

[1] A. Sen, "Descent Relations Among Bosonic D-branes," Int. J. Mod. Phys. A14 (1999) 4061, hep-th/9902105.

[2] A. Recknagel and V. Schomerus, "Boundary deformation theory and moduli spaces of D-branes," Nucl. Phys. B545 (1999) 233, hep-th/9811237;

C.G. Callan, I.R. Klebanov, A.W. Ludwig and J.M. Maldacena, "Exact solution of a boundary conformal field theory," Nucl. Phys. B422 (1994) 417, hep-th/9402113;

J. Polchinski and L. Thorlacius, "Free fermion representation of a boundary conformal field theory," Phys. Rev. D50 (1994) 622, hep-th/9404008.

[3] A. Sen, "Stable non-BPS bound states of BPS D-branes," JHEP 9808, (1998) 010, hep-th/9805019.

[4] A. Sen, "Tachyon condensation on the brane antibrane system," JHEP 9808 (1998) 012, hep-th/9805170.

[5] A. Sen, " $S O(32)$ spinors of type I and other solitons on brane-antibrane pait," JHEP 9809 (1998) 023, hep-th/9808141.

[6] E. Witten, "D-branes and K-theory," JHEP 12 (1998) 019, hep-th/9810188.

[7] A. Sen, "BPS D-branes on non-supersymmetric cycles," JHEP 12 (1998) 021, hepth/9812031.

[8] P. Horava, "Type IIA D-branes, K-theory and matrix theory," Adv. Theor. Math. Phys. 2 (1999) 1373, hep-th/9812135.

[9] A. Sen and B. Zweibach, "Tachyon Condensation in String Field Theory," hepth/9912249.

[10] W. Taylor, "D-brane effective field theory from string field theory," hep-th/0001201.

[11] N. Moeller and W. Taylor, "Level truncation and the tachyon in open bosonic string field theory," hep-th/0002237.

[12] J.A. Harvey and P.Kraus, "D-branes as unstable lumps in bosonic open string field theory," hep-th/0002117.

[13] R. de Mello Koch, A. Jevicki, M. Mihailescu and R. Tatar, "Lumps and p-branes in open string field theory," hep-th/0003031.

[14] N. Berkovits, "The tachyon potential in open Neveu-Schwarz String Field Theory," hep-th/0001084.

[15] N. Berkovits, A. Sen and B. Zweibach, "Tachyon condensation in superstring field theory," hep-th/0002211.

[16] P. de Smet and J. Raeymaekers, "Level four approximation to the tachyon potential in superstring field theory," hep-th/0003220.

[17] A. Iqbal and A. Naqvi, "Tachyon condensation on a non-BPS D-brane," hepth/0004015. 
[18] N. Moeller, A. Sen and B. Zweibach, "D-branes as tachyon lumps in string field theory," hep-th/0005036.

[19] J.A. Harvey, P. Krauss, F. Larsen and E.J. Martinec, "D-branes and strings as noncommutative solitons," hep-th/0005031.

[20] E. Witten, "Noncommutative tachyons and string field theory," hep-th/0006071.

[21] A. Bogojevic, A. Jevicki and G. Meng, "Quartic Interactions in Superstring Field Theory," Brown preprint HET-672, 1988, available from KEK preprint library.

[22] V.A. Kostelecky and S. Samuel, "The static tachyon potential in the open bosonic string theory," Phys. Lett. B207 (1988) 169;

V.A. Kostelecky and R. Potting, "Expectation values, Lorentz Invariance and CPT in the open bosonic string," Phys. Lett. B381 (1996) 89, hep-th/9605088.

[23] J.A. Harvey, P. Kraus, F. Larsen and E.J. Martinec, "D-branes and strings as noncommutative solitons," hep-th/0005031;

K. Dasgupta, S. Mukhi and G. Rajesh, "Noncommutative tachyons," JHEP 0006 (2000) 022, hep-th/0005006;

R. Gopakumar, S. Minwalla and A. Strominger, "Symmetry Restoration and Tachyon Condensation in Open String Theory," hep-th/0007226.

[24] A. Sen, "Universality of the Tachyon Potential," JHEP 9912 027, hep-th/9911116.

[25] R. de Mello Koch and J.P. Rodrigues, to appear.

[26] D. Gross and A. Jevicki, "Operator formulation of interacting string field theory (I),(II)," Nucl. Phys. B283 (1987) 1, B287 (1987) 225;

E. Cremmer, A. Schwimmer and C. Thorn, "The vertex function in Wittens formulation of string field theory," Phys. Lett. B179 (1986) 57;

S. Samuel, "The physical and ghost vertices in Witten's string field theory," Phys. Lett. B181 (1986) 255;

A. LeClair, M.E. Peskin and C.R. Preitschopf, "String field theory on the conformal plane. 1. Kinematical Principles," Nucl. Phys. B317 (1989) 411; "String Field Theory on the conformal plane. 2. Generalized Gluing," Nucl. Phys. B317 (1989) 464.

[27] N. Moeller, "Codimension two lump solutions in string field theory and tachyonic theories," hep-th/0008101. 